Proc. 12th Workshop on Nuclear Dynamics

February 3-10, 1996

Snowbird, Utah

BNL -63059

CONF-960294--l

\title{
INTRIGUING CENTRALITY DEPENDENCE OF THE AU-AU SOURCE SIZE AT THE AGS
}

\author{
Mark. D. Baker, ${ }^{1}$ for Experiment E866 (The E802 Collaboration ${ }^{2}$ ) \\ ${ }^{1}$ Massachusetts Institute of Technology, Cambridge, MA 02139 \\ ${ }^{2}$ ANL-BNL-UCBerkeley-UCRiverside-Columbia \\ INS(Tokyo)-Kyoto-LLNL-MIT-NYU-Tokyo-Tsukube
}

\section{INTRODUCTION}

One of the main goals of high energy heavy ion physics is to establish the existence of a deconfined phase of nuclear matter - the quark-gluon plasma - at high temperatures or densities. One possible signature of such a phase transition, especially if it were first order, would be a larger source size or lifetime than a similar hadronic system. At current AGS energies, we attempt to form a quark-gluon plasma by achieving a high baryon density for a period of time in the center of the collision region ${ }^{[1]}$. For a given density threshold, the size of this high density region should be a strong function of the impact parameter: the more central the event, the larger the high density region. Therefore, one possible signature of a quark-gluon plasma would be a sudden change in system lifetime or size as a function of the centrality of the collision.

In this talk we present an intriguing effect which was not predicted for simple hadronic systems: a rapid increase of the HBT-measured source radius parameter for pion pairs with increasing centrality for $A u-A u$ collisions at a beam momentum of 11.45 A GeV/c on a fixed target. Experience has shown, however, that we must be cautious in our interpretation. A complete understanding of the collision dynamics at a given energy must be built up from several measurements and new, but conventional, hadronic explanations must be considered for such unexpected effects. More study is needed, therefore, before any strong conclusions can be reached.

\section{HBT "SOURCE SIZE" MEASUREMENT}

Two-pion correlation functions for bosons, called Hanbury-Brown Twiss (HBT) or Bose-Einstein correlations, provide information about the length and time scales which characterize the pion source. In the simplest cases, we can directly relate the correlation function to the fourier transform of the source distribution and therefore 
the rms geometric size and lifetime of the source. In practice, this simple interpretation is complicated by two effects: dynamical correlations and the mixing of space and time.

Dynamical correlations are correlations between the spacetime position of pion emission and the pion momentum. These lead to effective "coherence lengths": emission points that are too far apart spatially cannot easily generate pairs which are close in momentum, making the measured source size parameter smaller than the geometric source size. HBT correlations measure the shortest length scales available, not necessarily the geometric length scale in which we are interested. Some progress has been made in studying the effect of dynamical correlations both theoretically ${ }^{[2]}$ and experimentally ${ }^{[3,4]}$, but we will ignore them for the purposes of this talk. Since we are interested in radius changes rather than absolute sizes, and since dynamical correlations will tend to wash out any interesting geometric effect rather than cause a centrality dependence, we are probably justified in ignoring them for now.

The mixing of space and time occurs because most of the HBT "source size" fit parameters which we can measure involve a mixture of the space and time length scales in the reference frame of interest: the collision center-of-mass frame. In general, this means that the "source duration of emission" fit parameter, $\tau$, is hard to extract. In some fit forms, this manifests itself as a poor phase-space coverage of the correlation function in the $\tau$ direction. In others, it manifests itself as $\tau$ being the difference, in quadrature, between large numbers.

Even in the presence of these complications, we should be able to extract useful information. The one-dimensional variable $Q_{R=r}$, defined as $\sqrt{|\vec{q}|^{2}+q_{0}^{2}}$, is conjugate to a quantity $R_{R=\tau}$ which has a well understood mixture of space and time scales:

$$
R_{R=\tau}=\sqrt{\frac{R^{2}+\tau^{2}\left\langle\beta_{\pi \pi}^{2} \cos ^{2} \theta\right\rangle}{1+\left\langle\beta_{\pi \pi}^{2} \cos ^{2} \theta\right\rangle}} \approx \sqrt{\frac{R^{2}+\tau^{2}}{2}},
$$

where $R$ is the gaussian-equivalent radius parameter, $\tau$ is the gaussian-equivalent emission duration, $\beta_{\pi \pi}$ is the pair velocity given by $\left(\vec{p}_{1}+\vec{p}_{2}\right) /\left(E_{1}+E_{2}\right)$, and $\theta$ is the angle between $\vec{q}=\vec{p}_{1}-\vec{p}_{2}$ and $\vec{\beta}_{\pi \pi}$. In our spectrometer acceptance, $\left\langle\beta_{\pi \pi}^{2} \cos ^{2} \theta\right\rangle \sim 1$. Therefore, we can control the mixing of $R$ and $\tau$ by fitting the correlation function to a gaussian in $Q_{R=\tau}: 1+\lambda \exp \left\{-Q_{R=\tau}^{2} R_{R=\tau}^{2}\right\}$. Interesting physics could show up as an increased geometric size or a longer lifetime; $R_{R=\tau}$ is sensitive to either or both signals.

For this particular measurement - pion pairs near mid-rapidity in a symmetric collision - the collision CM frame is the same as the longitudinal comoving system frame and we are not plagued by questions of which frame to use ${ }^{[b]}$. This means that the one-dimensional fit parameter $R_{R=r}$ can provide us with much of the information contained in the more sophisticated multi-dimensional fits without requiring as many pion pairs. The more common one-dimensional fit parameter $R_{\text {inv }}$, conjugate to $Q_{i n v} \equiv \sqrt{|\vec{q}|^{2}-q_{0}^{2}}$, is much more difficult to interpret.

\section{APPARATUS}

Experiment 866 at the BNL AGS is a fixed target experiment with a two-arm spectrometer ${ }^{[6,7]}$. Most of the data presented here are from the $1992 \mathrm{Au}$ beam when only one spectrometer (the wide-angle Henry Higgins), configured as in E859, was used. The data discussed in this talk were taken with the spectrometer at the " $21^{\text {on }}$ setting. Only negatively charged particles with momenta below $1.8 \mathrm{GeV} / \mathrm{c}$ which were identified 
as pions by the time-of-flight detector were considered for this analysis. The acceptance for negative pions at this setting is shown in Figure 1 . The data set consists of about 90000 negatively charged pion pairs.

We measure the violence of the collision using a zero-degree hadronic calorimeter (ZCAL) which measures the hadronic energy in roughly a forward cone $\theta_{\text {lab }}<1.5^{\circ}$. The fragments from the spectator breakup should be mostly contained within the ZCAL, so the ZCAL energy is proportional to the number of projectile spectator nucleons. From the number of projectile spectators, we can easily find the number of projectile participants $\left(N_{p p}\right)$. We expect the violence of the collision, as measured by $N_{p p}$, to be correlated with the impact parameter of the collision: the more central the event, the larger the $N_{p p}$.

\section{RESULTS}

For central events, the length scale (e.g. radius) of the initial collision region should be proportional to $N_{p p}^{1 / 3}$ since the volume is proportional to $N_{p p}$. Previous measurements of HBT radii have always yielded results which scaled roughly linearly with $N_{p p}^{1 / 3}$. Furthermore, the slopes have always been gentle in the sense that straight line fits through the data, $R=a+b N_{p p}^{1 / 3}$, have yielded intercept values, $a$, greater than or equal to zero[7,8].

Figure 2 shows the centrality dependence of the gaussian $R_{R=\tau}$ fit parameter from pion pairs near midrapidity for $A u-A u$ collisions from E866. The measured results cover a span of about $30 \%$ of the full spectator energy range available from the collision or the most central $15 \%$ of the cross-section. It should be noted that we are operating the ZCAL in an energy range where it is known to behave linearly and that the bin width is large compared to the resolution $(3-5 \sigma)$. Figure $2 b$ shows the dependence of $R_{R=\tau}$ on the calculated quantity $N_{p p}^{1 / 3}$. The dashed line $R_{R=\tau}=1.2 \mathrm{fm} \cdot N_{p p}^{1 / 3} / \sqrt{5}$ is the expected value for $R_{R=\tau}$ based on the transverse size of the original interaction region using hard-sphere geometry and assuming that $R_{R=\tau}=R_{L}=R_{T}=\tau$. The factor of $\sqrt{5}=\sqrt{3} \cdot \sqrt{5 / 3}$ comes from converting a hard sphere value to the gaussian form used in the fit, given the same rms. In the data, the growth of $R_{R=\tau}$ with centrality is steeper than expected, rising $40 \%$ in $R$ while $N_{p p}^{1 / 3}$ rises only by $7 \%$. Furthermore, the measured source size for the most central collisions is larger than the original interaction region (assuming $R_{L}, \tau \leq R$ as indicated in Refs. [7, 8]). Given the presence of dynamical correlations, the true source size might be even larger.

One possible mundane explanation for this effect is that we are seeing a pion freezeout radius and that the number of pions per participant is larger for central collisions due to secondary interactions. Preliminary measurements of the centrality dependence of the pion yield ${ }^{[9]}$ are not yet conclusive, but the dependence does not appear to be strong enough to cause such a steep change in the radius. Further studies are underway ${ }^{[10]}$. It is still possible that some smaller secondary effect coupled with a statistically unlikely fluctuation has caused this steep rise in the data.

\section{SYSTEMATIC CONSIDERATIONS}

As we pointed out above, it is difficult to understand how dynamical correlations alone could cause a rapid apparent growth of $R_{R=\tau}$, but we must be careful. If the $m_{T}$ 
Accepted phase space

(Figure adapted from Reference [7])

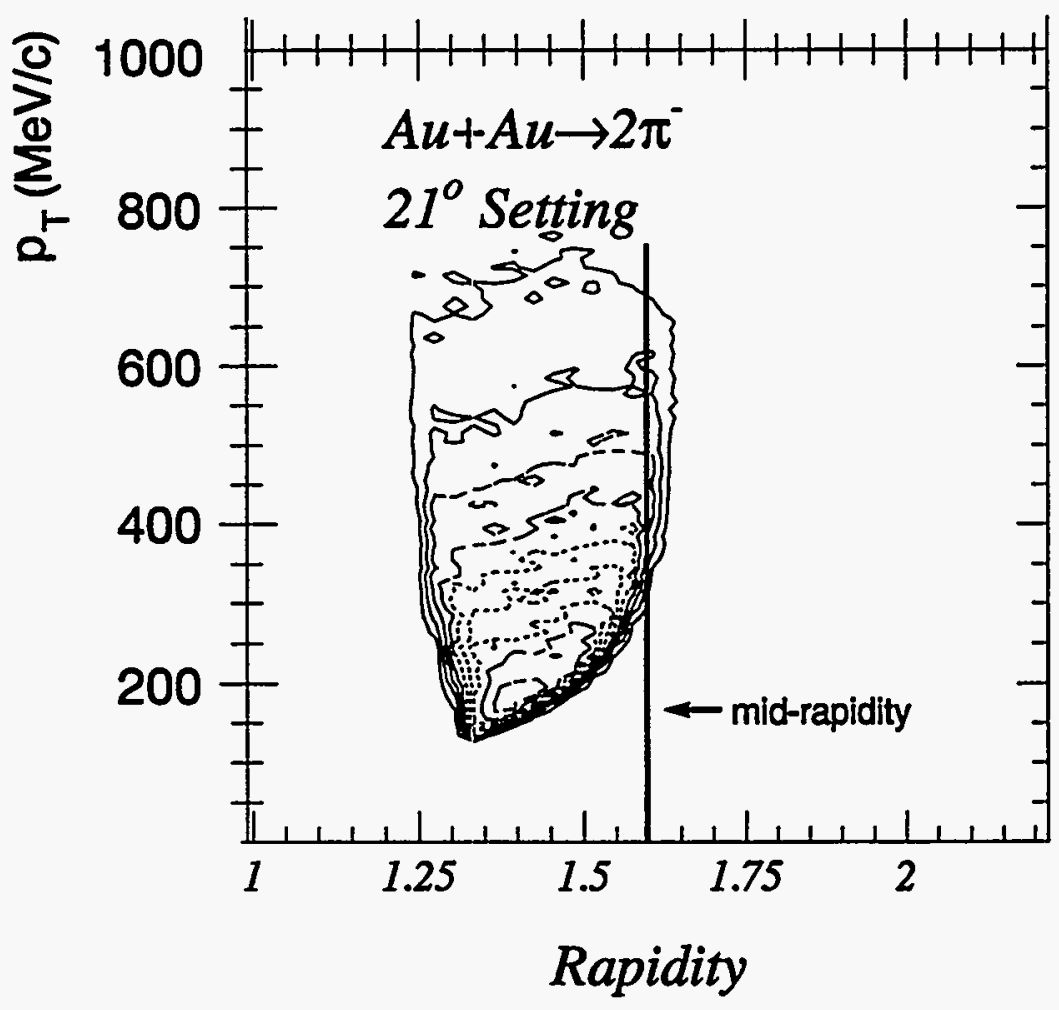


$A U+A U$ (1992) RESULTS NEAR $Y=Y_{c m}$

- $19922 \pi^{-}$trigger - negatives only - E866 - PRELIMINARY

* 1992 mixed trigger - E866 - VERY PRELIMINARY
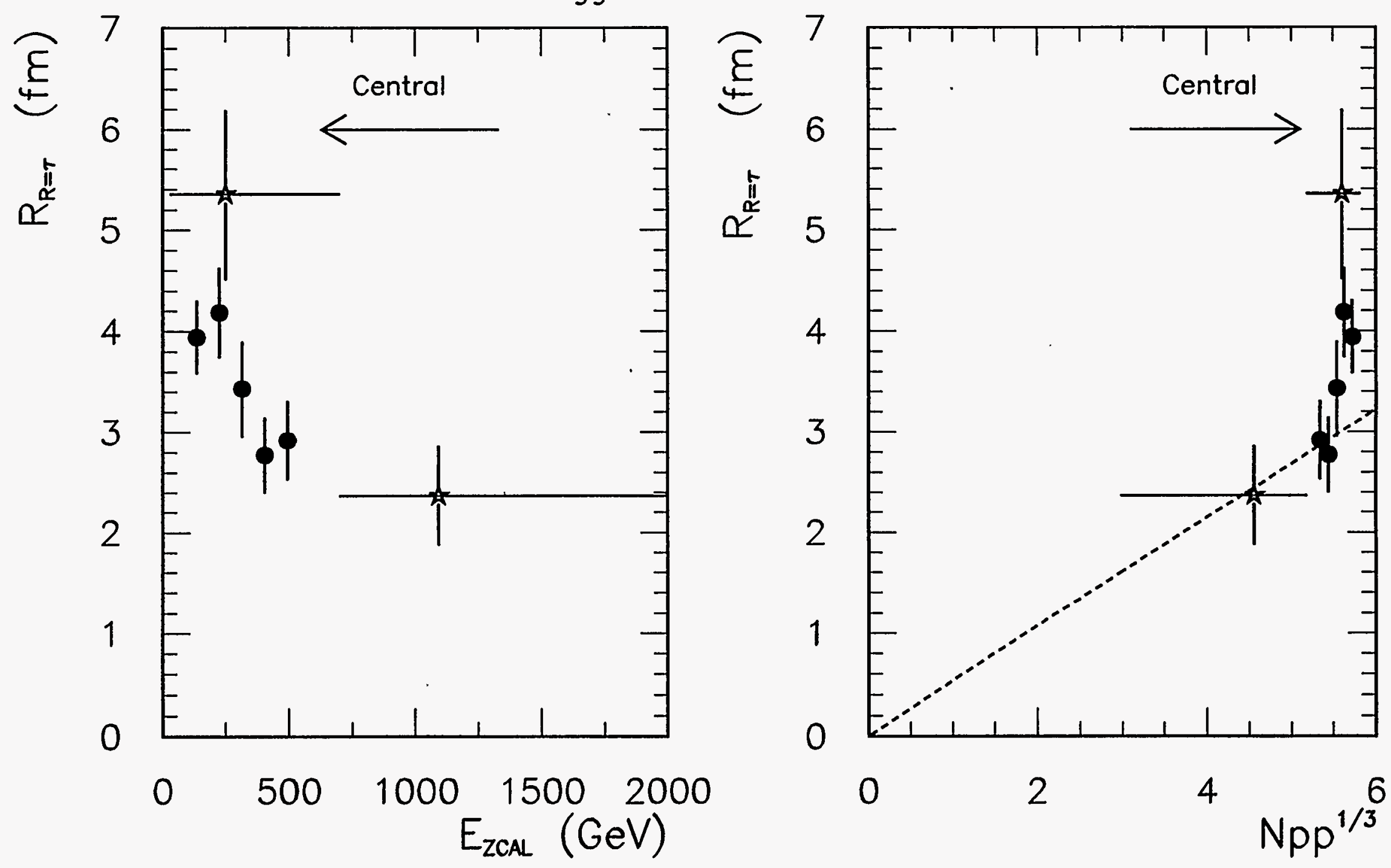


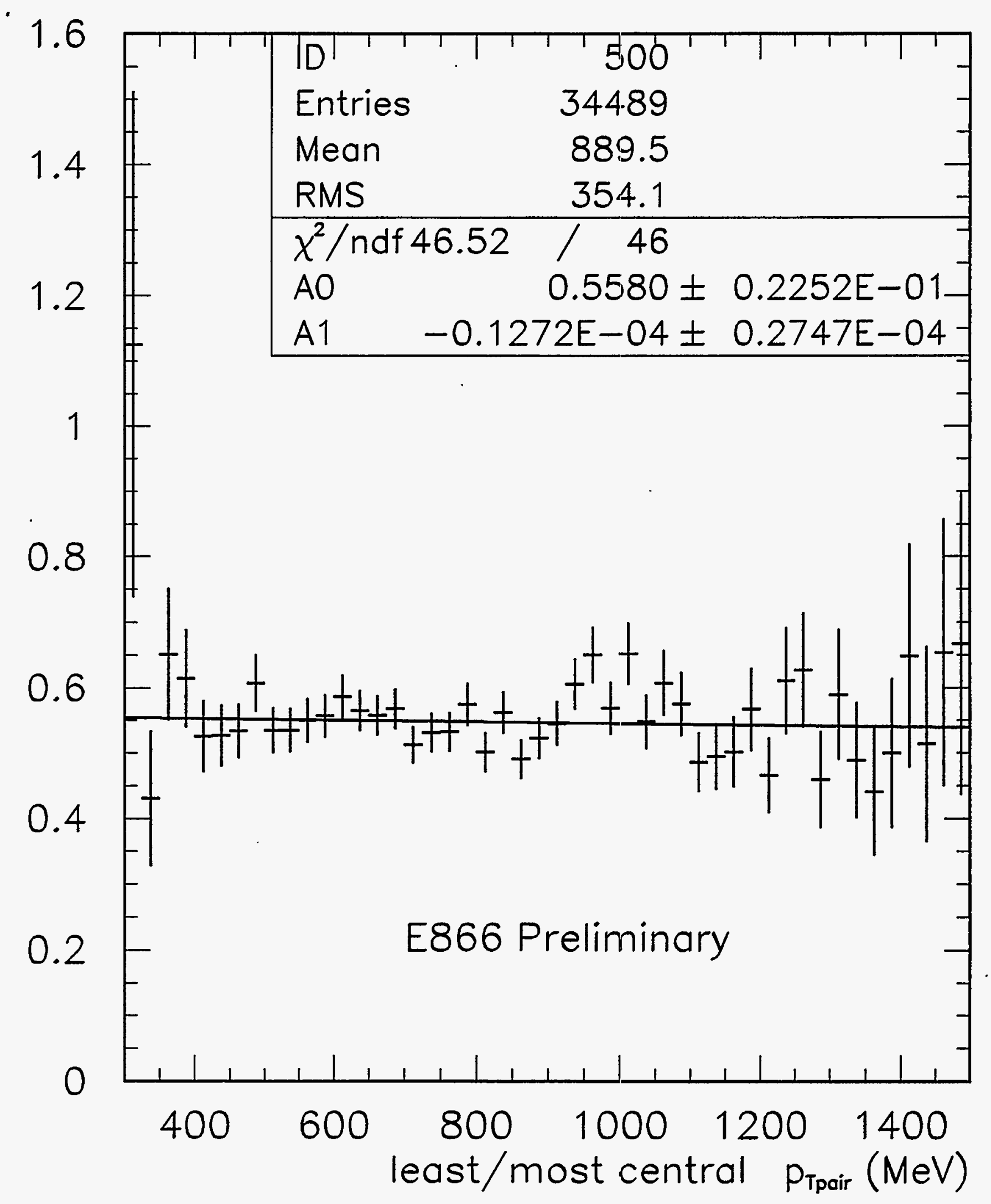


Figure 1. The acceptance for negative pions in the E866 apparatus for the data set discussed in this talk.

Figure 2. The centrality dependence of $R_{R=\tau}$ (a) vs. ZCAL Energy, (b) vs. $N_{p p}^{1 / 3}$. The colid points are from the $2 \pi^{-}$data set described in the text. The open stars are from a related dats set with a mixture of $2 \pi^{+}$and $2 \pi^{-}$data taken at $24^{\circ}$. The horisontal error bars show the bin sires, the vertical error bars show the error on the fit parameters, and the dashed line shows the simple geometric expectation based on the transverse sire of the original participant sone. 
Figure 8. The $p_{T}$ ratio for pairs between the most central and least central bin of the $2 \pi^{-}$data sample. The solid line is a straight line fit, showing a statistically negligible slope. The normalixation, which is related to the relative sample sise in the least and most central bin, is arbitrary.

distribution of the pion pairs softened with centrality, this would cause an apparent increase in the source size parameter with centrality since lower $m_{T}$ pairs tend to have a longer coherence length in the presence of dynamical correlations. Figure 3, however, shows that the $p_{T}\left(=\left|\left(\vec{p}_{1}+\vec{p}_{2}\right)_{T}\right|\right)$ distribution (and therefore the $m_{T}$ distribution) for accepted pairs is identical within errors between the most and least central bins discussed. Any small difference in the $m_{T}$ distribution allowed by the data would have a completely negligible effect on Figure 2.

We also performed another systematic test in order to validate the physics results. The test involved varying the binning and the two-particle cuts applied to the data and checking that the results did not change significantly. No significant variations were seen with cut changes or binning changes for any of the centrality bins individually or for the whole data set taken together. Also the $\chi^{2}$ values for all of the fits were reasonable. The success of this procedure gives us confidence in our handling of two-track efficiencies, in the lack of ghosts in our data, and in the stability of the fit procedure.

The final systematic consideration is the validity of the point-source Gamow correction which was applied to the data to correct for Coulomb effects before fitting. The point-source procedure has been compared to an iterative procedure which takes finite-size effects into account, leading to the conclusion that the point-source correction works well enough, especially for pions and for small data samples such as the one considered here ${ }^{[11]}$. 


\section{SUMMARY AND OUTLOOK}

We have observed a possible unpredicted, sharp rise in the variable $R_{R=\tau}$ versus centrality $\left(N_{p p}^{1 / 3}\right)$ for the most central events at AGS energies. The apparent source size is larger (or longer-lived) than the initial $A u$-Au overlap region. Further experimental study is needed to improve the statistical significance of the result and to determine whether it can be explained by conventional hadronic means or whether more exotic explanations can be admitted.

The E866 data set currently being analyzed contain millions of pion pairs which will allow us to examine multidimensional fits vs. both centrality and $m_{T p a i r}$. This data set also includes three global event characterization measurements: forward energy, multiplicity, and forward-particle reaction plane. Furthermore these pion pairs will cover a broad range in $N_{p p}$. This data set should allow us to understand the origin of the intriguing rise in $R_{R=\tau}$ with centrality.

\section{REFERENCES}

1. S. Kahana ef al., Searching for high baryon density at the AGS with ARC, Nucl. Phya. A.566:465c (1994).

2. U.A. Wiedemann, P. Scotto, and U. Heins, Transverse momentum dependence of HanburyBrown-Twiss correlation radii, PREPRINT TPR-95-06 (1995).

3. T. Alber, Two-pion interferometry in central nucleus-nucleus collisions at the CERN SPS results forom experiments NA35 and NA49, Nucl. Phys. A580:453c (1995).

4. B. Jacak, et al., Recent results from NA44 and a review of HBT, Nucl. Phys. A590:215c (1995).

5. S. Chapman, R. Nix, U. Heins, Extracting source parameters from gaussian fits to two particle correlations, Phys. Rev. C52:2694 (1895).

6. T. Abbott et al., NIM A 290 (1990) 41.

7. R.A. Solts. "Two-pion Correlation Measurements for $14.6 \mathrm{~A} \mathrm{GeV} / \mathrm{c} \mathrm{Si}+\mathrm{X}$ and $11.6 \mathrm{~A} \mathrm{GeV} / \mathrm{c}$ $\mathrm{An}+\mathrm{Au}$ " PhD thesis, MIT (1984).

8. B. Cole, et al., Recent results from experiment 859 at the BNL AGS, Nucl. Phys. A590:179e (1995).

9. D. Zachary. PhD thesis, MIT (1984).

10. Fuqiang Wang. $\mathrm{PhD}$ thesis, Columbia U. (in preparation).

11. V. Cienciolo. "2 $\mathrm{K}^{+}$Correlation Measurement" PhD thesis, MIT (1994).

\section{ACKNOWLEDGEMENTS}

The author would like to thank Ray Nix and Günther Roland for their comments on HBT. In addition, several members of the "E802 collaboration" contributed code or advice to the analysis that led to this talk. Vince Cianciolo, James Dunlop, Craig Ogilvie, and Ron Soltz made the most extensive contributions. Further advice came from: Larry Ahle, Chellis Chasman, Brian Cole, Lou Remsberg, Steve Steadman, George Stephans, Mike Tannenbaum, Fuqiang Wang, and Bill Zajc.

Experiment 866 is supported by the U.S. Department of Energy (ANL, BNL, UCBerkeley, UC-Riverside, Columbia, LLNL, and MIT), by NASA (UC-Berkeley), and by the US-Japan High Energy Physics Collaboration treaty. 


\section{DISCLAIMER}

This report was prepared as an account of work sponsored by an agency of the United States Government. Neither the United States Government nor any agency thereof, nor any of their employees, makes any warranty, express or implied, or assumes any legal liability or responsibility for the accuracy, completeness, or usefulness of any information, apparatus, product, or process disclosed, or represents that its use would not infringe privately owned rights. Reference herein to any specific commercial product, process, or service by trade name, trademark, manufacturer, or otherwise does not necessarily constitute or imply its endorsement, recommendation, or favoring by the United States Government or any agency thereof. The views and opinions of authors expressed herein do not necessarily state or reflect those of the United States Government or any agency thereof. 